\title{
Spatial Curvature and Large Scale Lorentz Violation
}

\author{
Jing Li ${ }^{a}$, Yongxiang Zhou ${ }^{a}$ and Xun Xue ${ }^{a, b}, 1$ \\ ${ }^{a}$ Department of Physics, East China Normal University, Shanghai 200241, China \\ ${ }^{b}$ Center for Theoretical Physics, Xinjiang University, Urumqi 830046, China
}

\begin{abstract}
The tension between the Hubble constant obtained from the local measurements and from cosmic microwave background (CMB) measurements motivated us to consider the cosmological model beyond $\Lambda \mathrm{CDM}$ one. We investigate the cosmology in the large scale Lorentz violation model with non-vanishing spatial curvature. The degeneracy among spatial curvature, cosmological constant and cosmological contortion distribution makes the model viable in describing the known observation date. We get some constraints on the spatial curvature by the comparison of the relation between measured distance modulus and red-shift with the predicted one, the evolution of matter density over time and the evolution of effective cosmological constant. The performance of large scale Lorentz violation model with non-vanishing spatial curvature under these constrains is discussed.
\end{abstract}

\section{Introduction}

The present Hubble constant value can be measured in many ways, e.g., by the $\Lambda$ CDM model with CMB measurement fixing its parameters at relatively early universe, or by the distance ladder method at relatively late universe. The discrepancy between the values of these different methods is known as the $H_{0}$ tension problem.

In the late 1990s the use of Type Ia supernovae as standard candles led to the discovery that the expansion of the universe is accelerating. Large-scale observations indicates a phase of cosmological acceleration which occurs at late time 1]. Hence, several attempts have been proposed to describe the cosmic accelerated scenario. In general, there are two kinds of interpretations for this cosmic phase: i) postulating an exotic form of energy with negative pressure usually called dark energy or ii) modifying the laws of gravity. Numerous models have been proposed based on these two branches, but it is difficult to determine which one is correct due to the degeneracies in the parameter space. Despite this, the $\Lambda$ CDM model with six parameters could excellently fit almost all observational data, and has been set as the standard model of cosmology 2]. By assuming flat space in $\Lambda \mathrm{CDM}$ model, the final full-mission Planck measurements of the cosmic microwave background (CMB) anisotropies has found a value of the Hubble constant $H_{0}=(67.27 \pm 0.60) \mathrm{km} / \mathrm{s} / \mathrm{Mpc}$. This is compatible with many earlier and recent estimates of $H_{0}$. In contrast, multiple local expansion rate measurements find slightly higher $H_{0}$ values and slightly larger error bars. And the latest value from the Supernovae and $H_{0}$ for the Dark Energy Equation of State $\left(\mathrm{S} H_{0} \mathrm{ES}\right)$ project together with GAIA DR2 parallaxes is $H_{0}=(73.52 \pm 1.62) \mathrm{km} / \mathrm{s} / \mathrm{Mpc}$, which has a more than $3 \sigma$ tension with the Planck CMB data 3 . This tension is one of the most intriguing problems in modern cosmology. There have been many attempts to solve the problem, such as introducing new physics beyond the standard $\Lambda \mathrm{CDM}$ cosmological model. The non-zero spatial curvature may be an choice of solution to the $H_{0}$ tension problem. Observing that the Friedmann equation $\dot{a}^{2}+K=\frac{8 \pi G}{3} \rho a^{2}$ $\Lambda \mathrm{CDM}$ model with spatial curvature can be written as

$$
1-\frac{1}{\Omega}=\frac{3 K}{8 \pi G \rho a^{2}}
$$

where $\Omega=\frac{\rho}{\rho_{c}}=\frac{8 \pi G \rho}{3}\left(\frac{a}{\dot{a}}\right)^{2}$ is cosmic density, one can find $\frac{3 K}{8 \pi G \rho a^{2}} \propto a^{2}$ in the radiation dominated era while $\frac{3 K}{8 \pi G \rho a^{2}} \propto a$ in the matter-dominated era for $\rho a^{3}=$ const then. In a word, the absolute

\footnotetext{
${ }^{1}$ Corresponding author:xxue@phy.ecnu.edu.cn
} 
value of $1-\frac{1}{\Omega}$ should grow all the time from the early universe until now if $K \neq 0$ because the cosmic scale factor is growing all the time. From the Planck period until now, $a(t)$ has increased by dozens of magnitudes, then the value of $1-\frac{1}{\Omega}$ has also increased by dozens of magnitudes. Nowadays, the deviation of $\Omega$ to 1 is observed to be a magnitude of up to 1 , then the current value of $1-\frac{1}{\Omega}$ should also be a magnitude of 1. Based on existing conclusions, we can get the cosmological density of the Planck period $\Omega_{p}=1 \pm 10^{-N}$, where $\mathrm{N}$ is a constant more than dozens. It suggests that at the end of the Planck period, the critical density and cosmic mass density were the same in many significant numbers, and the two physical quantity were not exactly equal. Otherwise, after the long evolution, there is a universe without the present spatial quasi-flatness. The non-zero spatial curvature encounters the problem of fine-tuning somehow in the inflation scenario. However it is still sensible to figure out whether the present distance observations prefer a non-zero spatial curvature or not. Although the current cosmological observations strongly favor a spatially flat Universe, e.g., the combined Planck 2018 cosmic microwave background (CMB) and baryon acoustic oscillation measurements, which suggest that $\Omega_{K}=0.001 \pm 0.002$ [1], these constraints, however, are based on the pre-assumption of a specific cosmological model (e.g., the standard $\Lambda \mathrm{CDM}$ model). Because of the strong degeneracy between spatial curvature and the equation of state of dark energy [4], it is rather difficult to constrain the two quantities simultaneously. In general, dark energy is assumed to be a cosmological constant for the estimation of curvature, or conversely, the Universe is assumed to be flat in a dark energy analysis. Indeed, if the density of dark energy is allowed to vary freely with time, constraints on the geometry of the Universe may not only become less stringent, but may even depend on the properties that dark energy is assumed to have at early times 5 . But a simple flatness assumption may result in an incorrect reconstruction of the dark energy equation of state, even if the real curvature is very tiny, and a cosmological constant assumption may lead to confusion between $\Lambda \mathrm{CDM}$ and a dynamical dark energy model[6]. Moreover, the tension between the most recent SneIa data and spatial flat assumption in the $\Lambda \mathrm{CDM}$ model[7 may eventually support a spatial non-flat universe 8 . It is pointed out that the Planck $2018 \mathrm{CMB}$ spectra preferring a positive spatial curvature at more than 99\% confidence level(CL) 9 . The combined analysis of Planck CMB anisotropy and luminosity distant data simultaneously excludes a flat universe and a cosmological constant at 99\% CL[10].

The motivation behind the choice of non-flat cosmology is that, as usually believed, an early inflationary phase leads today to almost flat universe, albeit not exactly with a perfectly zero spatial curvature. This is not necessary if the number of e-foldings is not very large 11]. It is still possible that there is a contribution to the Friedmann equations from the spatial curvature when studying latetime universe, though much smaller than other energy components according to observations. Recent observations have complicated the problem by suggesting that the deceleration factors of the expansion of the universe change over time, and that the universe went from decelerating to accelerated expansion about 6 billion years ago.

In the $\Lambda \mathrm{CDM}$ model, the cosmological constant can be viewed as from vacuum energy density, which is responsible for the accelerated expansion of the universe. Considering the large scale Lorentz violation, the large-scale torsion distribution caused by the Lorentz violation effect combined with the vacuum energy density is responsible for the accelerating expansion[12]. Therefor we can introduce the effective cosmological constant which is responsible for the accelerating expansion of the universe. Using the evolution of matter density $\Omega_{M}$ and initial conditions to constrain the bare cosmological constant to a desirable range. The performance of large scale Lorentz violation model with non-vanishing spatial curvature under these constrains will be discussed in this paper .

\section{Cosmology of the Gravitation Theory with Large Scale Lorentz Violation}

The large scale Lorentz violation gravitation model with zero spatial curvature is discussed in Reference [12. The general FRW metric including the open and closed spatial geometry is

$$
d s^{2}=d t^{2}-a^{2}(t)\left(d r^{2} /\left(1-K r^{2}\right)+r^{2} d \theta^{2}+r^{2} d \theta^{2}+r^{2} \sin ^{2} \theta d \varphi^{2}\right) .
$$

The equations of motion for gravitational field equation is

$$
\tilde{G}^{a}{ }_{b}=\tilde{R}_{b}^{a}{ }_{b}-1 / 2 \tilde{R} \delta^{a}{ }_{b}=8 \pi G / c^{4}\left(T_{M}+T_{\Lambda}\right)^{a}{ }_{b},
$$


in which $\tilde{G}^{a}{ }_{b}, \tilde{R}^{a}{ }_{b}$ and $\tilde{R}$ are respectively the Einstein tensor, Ricci curvature tensor and Ricci curvature scalar of the spacetime with Levi-Civita connection. $T_{\Lambda}$ is energy-momentum tensor of the dark partner contributed by the contortion tensor, which is responsible for the accelerated expansion of the universe, $\left[T_{\Lambda}\right]_{b}^{a}=\operatorname{diag}\left(\rho_{\Lambda},-p_{\Lambda},-p_{\Lambda},-p_{\Lambda}\right)$. The dark partner energy density $\rho_{\Lambda}$ and pressure $p_{\Lambda}$ are expressed as

$$
\begin{aligned}
\rho_{\Lambda} & =-\frac{c^{4}}{8 \pi G}\left(3 \mathcal{K}^{2}+6 \mathcal{K} \frac{\dot{a}}{a}-\Lambda_{0}\right) \\
p_{\Lambda} & =-\frac{c^{4}}{8 \pi G}\left(\mathcal{K}^{2}+4 \mathcal{K} \frac{\dot{a}}{a}+2 \dot{\mathcal{K}}-\Lambda_{0}\right)
\end{aligned}
$$

where $\mathcal{K}(t)=K_{11}^{0}=K_{22}^{0}=K_{33}^{0}$ is the non-zero components of the contortion tensor and $\Lambda_{0}$ is the bare cosmological constant, the geometrical contribution by vacuum energy density. The modified Friedmann Equation in geometrical unit $\frac{c^{4}}{8 \pi G}=1$ can be written as

$$
\left(\frac{\dot{a}}{a}\right)^{2}+2 \frac{\dot{a}}{a} \mathcal{K}+\mathcal{K}^{2}+\frac{K}{a^{2}}=\frac{1}{3}\left(\rho+\Lambda_{0}\right)
$$

and

$$
\ddot{a}=-\frac{a}{2}\left(p+\frac{1}{3} \rho\right)+\frac{1}{3} a \Lambda_{0}-\frac{d}{d t}(a \mathcal{K})
$$

This paper aims to explore the performance of the large scale Lorentz violation model with non-vanishing spatial curvature. It should be noted that the model lacks the evolution of $\mathcal{K}(t)$, the set of equations (6) and (7) is not closed. As discussed in 12 14, the scales of Lorentz violation region during quantum gravity dominating era experience the stretching out beyond the horizon by inflation and the region with sub-scale may reenter the horizon during normal expansion. In principle it is needed the specific quantum gravity model and inflation model to give the prediction on the evolution of $\mathcal{K}(t)$. However, the evolution of $\mathcal{K}(t)$ can be approximated by employing the $\Lambda$ CDM model or assuming an equation of state satisfied by the dark partner phenomenologically. One can add an independent equation from the set of Friedmann equations of the $\Lambda$ CDM model to close the set of modified Friedmann equations with two choices as in [13, 14]. The third approximation comes from adding the equation of state for the dark partner to the modified Friedmann equations set. The three kinds of approximation can be given as follows as in [13, 14.

The first kind of approximation named Case A is

$$
\frac{1}{3} a \Lambda_{0}-\frac{1}{3} a \Lambda=\frac{d}{d t}(a \mathcal{K})
$$

and the second kind named Case B is

$$
(3 w+2) \frac{\dot{a}}{a} \mathcal{K}+\dot{\mathcal{K}}+\frac{3 w+1}{2} \mathcal{K}^{2}+\frac{3 w+1}{2} \frac{K}{a^{2}}+\frac{w+1}{2}\left(\Lambda-\Lambda_{0}\right)=0,
$$

while the third one named Case $\mathrm{C}$ is

$$
\left(4+6 w_{0}\right) \frac{\dot{a}}{a} \mathcal{K}+\left(1+3 w_{0}\right) \mathcal{K}^{2}+2 \dot{\mathcal{K}}=\left(w_{0}+1\right) \Lambda_{0}
$$

where $w$ is the equation of state parameter for cosmic media in $p=w \rho$ and $w_{0}$ is the equation of state parameter for dark partner part in $p_{\Lambda}=w_{0} \rho_{\Lambda}$.

The Friedmann equation of $\Lambda \mathrm{CDM}$ model can be written as

$$
\Lambda=3\left(\frac{\dot{a}}{a}\right)^{2}-\rho
$$

Similarly, the modified Friedmann equation of the large scale Lorentz violation model can be written as

$$
\Lambda_{0}-6 \mathcal{K} \frac{\dot{a}}{a}-3 \mathcal{K}^{2}-3 \frac{K}{a^{2}}=3\left(\frac{\dot{a}}{a}\right)^{2}-\rho
$$


Comparing (11) and (12), it is not only the bare cosmological constant $\Lambda_{0}$ that contributes to the accelerated expansion of the universe in the large scale Lorentz violation model, but also the contribution from $\mathcal{K}(t)$, whose total contribution can define the effective cosmological constant

$$
\Lambda_{e f f} \equiv \Lambda_{0}-6 H \mathcal{K}-3 \mathcal{K}^{2}-\frac{3 K}{a^{2}} .
$$

The initial value of $\mathcal{K}(t)$, the contortion component value at present time, can be obtained by the Friedmann equation of $\Lambda \mathrm{CDM}$ model with two possible choices. The first kind of initial value is

$$
\mathcal{K}\left(t_{0}\right)=H_{0}\left\{-1+\sqrt{1-\frac{K}{a_{0}^{2} H_{0}^{2}}-\frac{\Lambda}{3 H_{0}^{2}}+\frac{\Lambda_{0}}{3 H_{0}^{2}}}\right\}
$$

while the second one is

$$
\mathcal{K}\left(t_{0}\right)=H_{0}\left\{-1-\sqrt{1-\frac{K}{a_{0}^{2} H_{0}^{2}}-\frac{\Lambda}{3 H_{0}^{2}}+\frac{\Lambda_{0}}{3 H_{0}^{2}}}\right\} .
$$

To ensure the value of contortion components take real numbers, there should be a constrain condition satisfied by the bare cosmological constant, the cosmological constant observed and the spatial curvature, $K / a_{0}^{2}+\Lambda / 3-\Lambda_{0} / 3 \leq H_{0}^{2}$, i.e.

$$
\Lambda_{0} \geq \Lambda+\frac{3 K}{a_{0}^{2}}-3 H_{0}^{2} .
$$

For a specific large scale Lorentz violation model, the spatial curvature constant $K$, the current cosmic scale factor $a_{0}$, Hubble constant $H_{0}$ and the bare cosmological constant $\Lambda_{0}$ are the free input parameters given by observation or predicted by more fundamental theory model. It can be defined that

$$
\Lambda_{\min }=\Lambda+\frac{3 K}{a_{0}^{2}}-3 H_{0}^{2}
$$

from condition (16) so that there is a minimum for the free input bare cosmological constant, $\Lambda_{0} \geq \Lambda_{\text {min }}$, to give a reasonable evolution of the universe.

Since the accelerated expansion occurs in the late stage of the cosmic evolution, we mainly concentrate to consider the matter-dominated era. In this case of $w=0$, the evolution of the Hubble parameters and contortion can be obtained by the modified Friedmann equations $(6,,(7)$ and one of the three kind of approximations, (8), (9) and (10), with both the initial values of $\mathcal{K}(t)$ in (14) and (15). The evolution of $H$ versus time in different cases are presented in Figures 1,2 and 3 . Figures 1 show that the behavior of the evolution of Hubble parameter of Case $\mathrm{C} 2$ deviates relatively large from other cases and the $\Lambda \mathrm{CDM}$ model. However, deviation among different models become small after the scale factor of the universe, $a$, get greater than 1 . The evolution of Case $\mathrm{B}$ and $\Lambda \mathrm{CDM}$ models tends to be identically the same globally.

Figures 2 and 3 show that the evolution of Case A2 and Case C2 in the case of $K=-1$ deviates from the behavior of the $\Lambda \mathrm{CDM}$ model, while the deviations among Case A1, Case B1, Case C1, Case B2 and $\Lambda \mathrm{CDM}$ models are small. The Figures 1,2 and 3 show that the evolution in three kind of approximation respectively is very close to the evolution in the $\Lambda \mathrm{CDM}$ model when $K=-1$ and $\mathcal{K}(t)$ takes the first initial value. Since the light ray travels along the geodesic line even in the case of non-zero torsion, the redshift formula for the large scale Lorentz violation model is the same as for the $\Lambda$ CDM model,

$$
1+z=\frac{a_{0}}{a} .
$$

From definition of luminosity distance, one can get

$$
d_{L}(z)=(1+z) \frac{d_{H}}{\sqrt{\Omega_{K}}} \sinh \left[\sqrt{\Omega_{K}} \int_{0}^{z} \frac{H_{0}}{H\left(z^{\prime}\right)} d z^{\prime}\right],
$$

where $d_{L}$ is the luminosity distance,

$$
d_{H}=\frac{c}{H_{0}}
$$




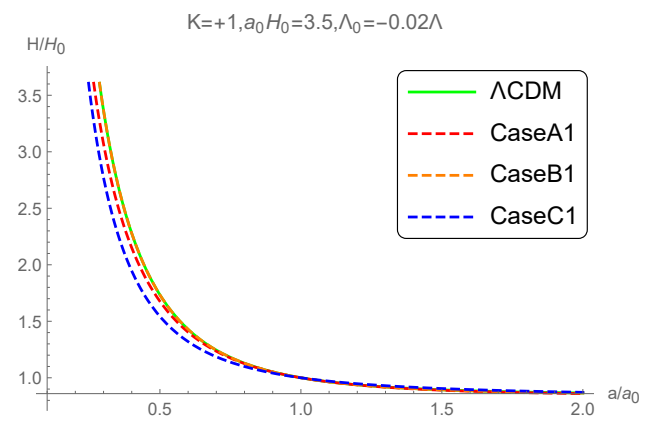

(a)

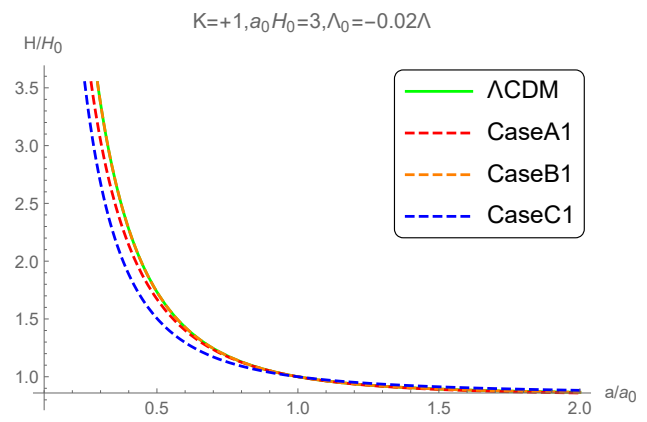

(c)

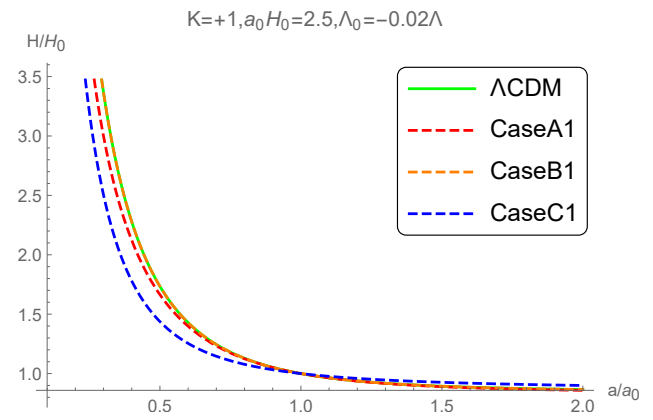

(e)

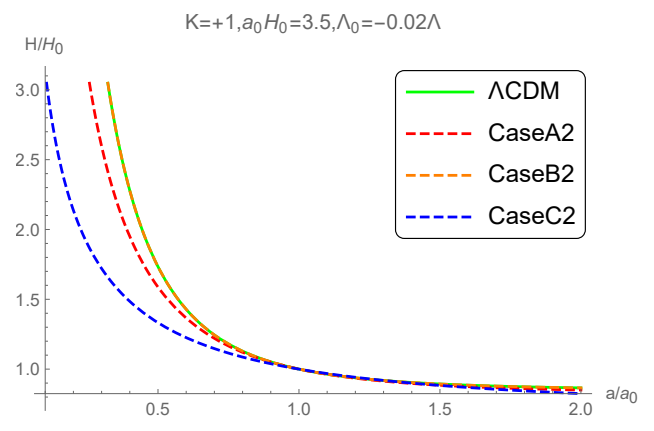

(b)

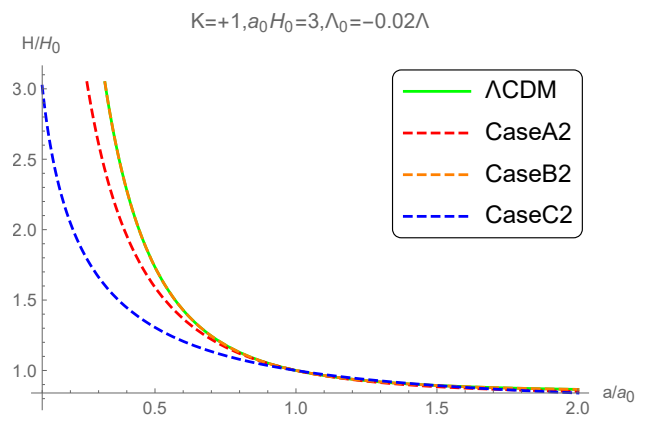

(d)

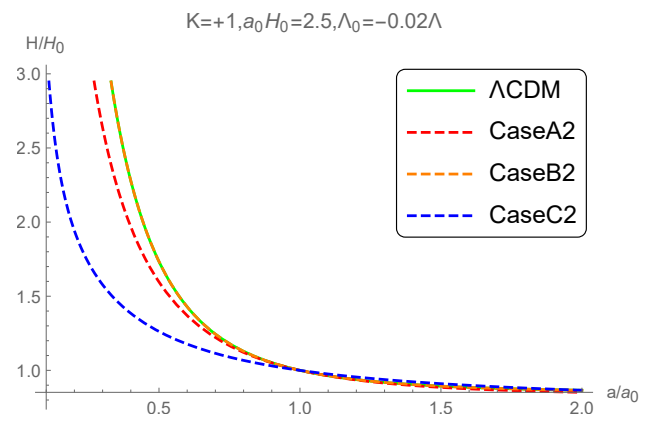

(f)

Figure 1: The Hubble constant evolves with the scale factor when $K=+1, \Lambda_{0}=-0.02 \Lambda$.

the Hubble distance and

$$
\Omega_{K}=-\frac{K}{{H_{0}}^{2} a_{0}{ }^{2}}
$$

is the curvature density at present. One can derive the set of equations for $\mathcal{K}(z)$ and $d_{L}(z)$ with formula (19),

$$
\begin{aligned}
& \frac{d_{L}{ }^{\prime \prime}(z)(1+z)^{6}\left(1-\frac{K d_{L}{ }^{2}(z)}{2 a_{0}{ }^{2}(1+z)^{2}}\right)^{2}}{\left[d_{L}{ }^{\prime}(z)(1+z)-d_{L}(z)\right]^{3}}-\frac{1}{2} \frac{(1+z)^{4}\left(1-\frac{K d_{L}{ }^{2}(z)}{2 a_{0}{ }^{2}(1+z)^{2}}\right)^{2}}{\left[d_{L}{ }^{\prime}(z)(1+z)-d_{L}(z)\right]^{2}}+\frac{K}{a_{0}{ }^{2}} \frac{d_{L}(z)(1+z)^{2}\left(1-\frac{K d_{L}{ }^{2}(z)}{2 a_{0}{ }^{2}(1+z)^{2}}\right)}{d_{L}{ }^{\prime}(z)(1+z)-d_{L}(z)} \\
& +2 \frac{(1+z)^{2}\left(1-\frac{K d_{L}{ }^{2}(z)}{2 a_{0}{ }^{2}(1+z)^{2}}\right)}{d_{L}{ }^{\prime}(z)(1+z)-d_{L}(z)} \mathcal{K}(z)-\frac{(1+z)^{3}\left(1-\frac{K d_{L}{ }^{2}(z)}{2 a_{0}{ }^{2}(1+z)^{2}}\right)}{d_{L}{ }^{\prime}(z)(1+z)-d_{L}(z)} \mathcal{K}^{\prime}(z)+\frac{1}{2} \mathcal{K}^{2}(z)+\frac{K}{2 a^{2}}-\frac{1}{2} \Lambda_{0}=0, \quad(22)
\end{aligned}
$$




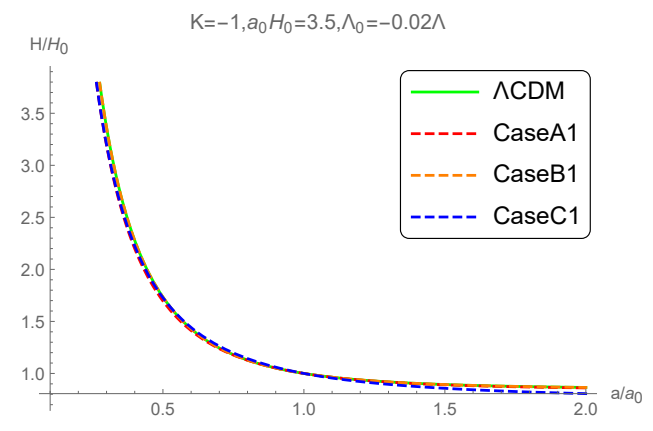

(a)

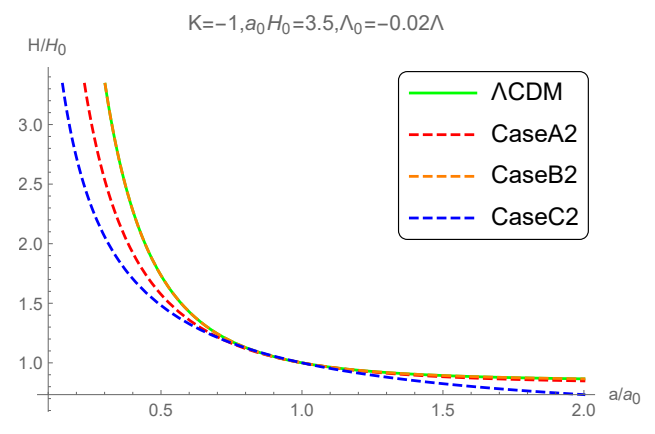

(b)

Figure 2: The Hubble constant evolves with the scale factor when $K=-1, \Lambda_{0}=-0.02 \Lambda, a_{0} H_{0}=3.5$.

together with the Case A,

$$
\frac{(1+z)^{2}\left(1-\frac{K d_{L}^{2}(z)}{2 a_{0}{ }^{2}(1+z)^{2}}\right)}{d_{L}{ }^{\prime}(z)(1+z)-d_{L}(z)}\left[\mathcal{K}(z)-(1+z) \mathcal{K}^{\prime}(z)\right]=\frac{1}{3}\left(\Lambda_{0}-\Lambda\right),
$$

the Case B,

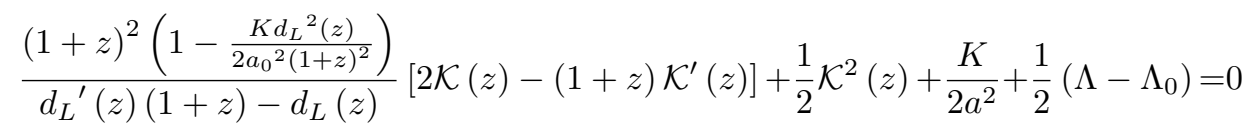

and the Case C,

$$
\frac{(1+z)^{2}\left(1-\frac{K d_{L}{ }^{2}(z)}{2 a_{0}^{2}(1+z)^{2}}\right)}{d_{L}{ }^{\prime}(z)(1+z)-d_{L}(z)}\left[\mathcal{K}(z)+(1+z) \mathcal{K}^{\prime}(z)\right]=-\mathcal{K}^{2}(z),
$$

respectively. The distance modulus defined by

$$
\mu=25+5 \log \left(\frac{d_{\mathrm{L}}}{M \mathrm{pc}}\right)
$$

is often used in astronomical observation. Figures 4 show the evolution of the luminosity distance versus redshift.

\section{The Numerical Predictions}

The modified Friedmann equation (12) of the large scale Lorentz violation model can be written as

$$
\frac{\rho}{3 H^{2}}+\frac{\Lambda_{0}}{3 H^{2}}-\frac{2 \mathcal{K}}{H}-\left(\frac{\mathcal{K}}{H}\right)^{2}-\frac{K}{a^{2} H^{2}}=1
$$

and

$$
\Omega_{M}+\Omega_{e f f}=1,
$$

where

$$
\Omega_{M}=\frac{\rho}{3 H^{2}}
$$

is the matter density, $\Omega_{e f f}=\frac{\Lambda_{0}}{3 H^{2}}-\frac{2 \mathcal{K}}{H}-\left(\frac{\mathcal{K}}{H}\right)^{2}-\frac{K}{a^{2} H^{2}}$ is the contribution of the bare cosmological constant, contortion and spatial curvature. The minimum value for $\Lambda_{0}$ in the case of $K=+1,-1$ is shown in Figure 5. Utilizing the modified Friedmann equation, for every kind of approximation models, one can obtain the evolution of the matter energy density with time. The evolution of $\Omega_{M}$ in the three kind of approximation models at different values of $\Lambda_{0}$ is shown in Figure 6. The $\Omega_{M}$ will decrease to be 


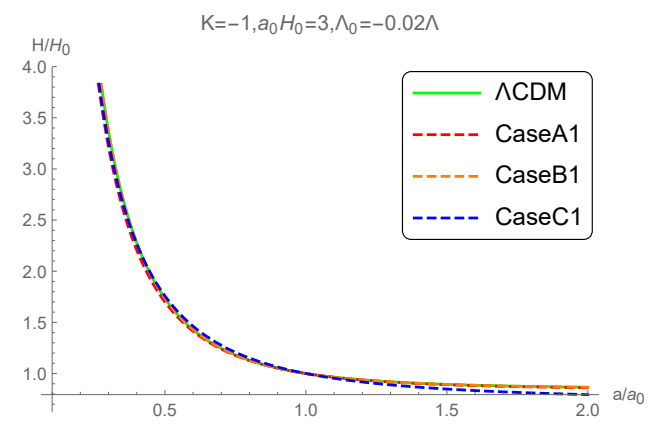

(a)

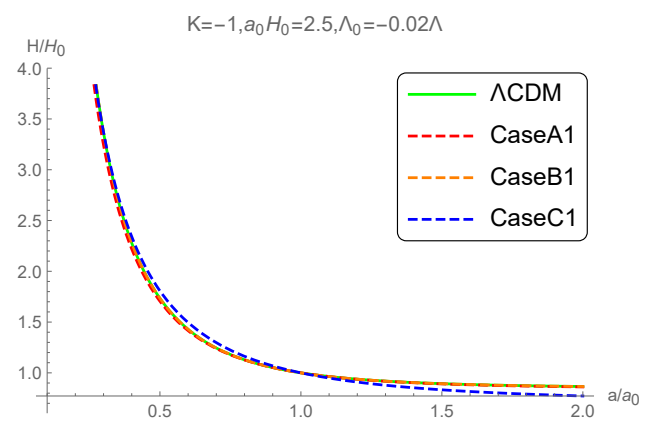

(c)

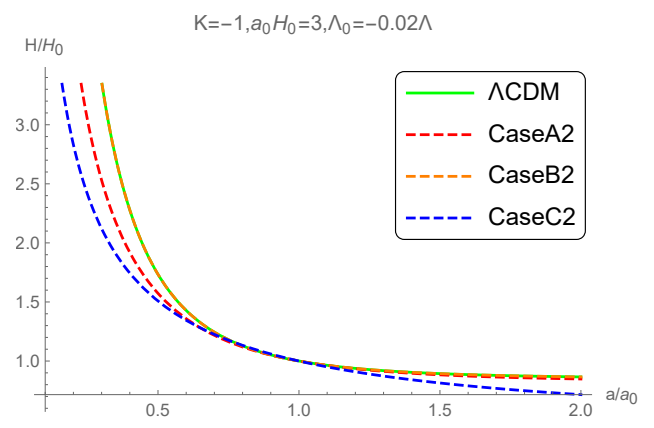

(b)

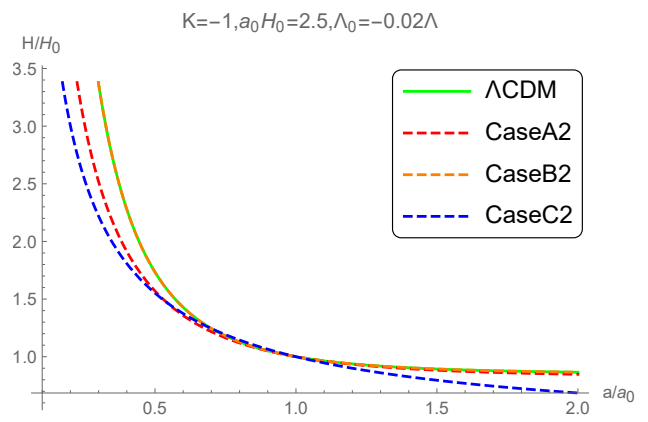

(d)

Figure 3: The Hubble constant evolves with the scale factor when $K=-1, \Lambda_{0}=-0.02 \Lambda, a_{0} H_{0}=3$ and 2.5

negative along with the increase of the $\Lambda_{0}$ beyond a critical value, $\Lambda_{\max }$. It leads to a constrain condition on the $\Lambda_{0}$ value,

$$
1-\frac{\Lambda_{0}}{3 H^{2}}+\frac{2 \mathcal{K}}{H}+\left(\frac{\mathcal{K}}{H}\right)^{2}+\frac{K}{a^{2} H^{2}} \geq 0,
$$

to ensure that $\Omega_{M} \geq 0$. Constrain 30 sets a maximum $\Lambda_{\max }$,

$$
\Lambda_{\max }=3 H^{2}+6 H \mathcal{K}+3 \mathcal{K}^{2}+\frac{3 K}{a^{2}},
$$

for the $\Lambda_{0}$ value. When $\Lambda_{0}>\Lambda_{\max }$, the value of $\Omega_{M}$ will be less than 0 . Table 1 and 2 show the $\Lambda_{\max }$ values for all the cases in consideration.

It can be observed that $\Omega_{M}$ will decrease to be negative whenever the value of $\Lambda_{0}$ is positive. So the reasonable values of $\Lambda_{0}$ should be non-positive, i.e. $\Lambda_{\max } \approx 0$. In 13, 14, the authors point out that there is a critical value for $\Lambda_{0}$, named as $\Lambda_{0-c r i t}$, which stands for the separation of two phases in the evolution pattern of $\Lambda_{\text {eff }}$ versus $t$, i.e. $\Lambda_{\text {eff }}$ decreases monotonically along with the increase of $t$ when $\Lambda_{0} \leq \Lambda_{0-c r i t}$ while its evolution has a local minimum when $\Lambda_{0}>\Lambda_{0-\text { crit }}$, and the $\Lambda_{0-\text { crit }}$ is approximately zero. In other words, $\Lambda_{\text {eff }}$ is monotonically decreasing when $\Lambda_{0}$ is from the string landscape, while $\Lambda_{\text {eff }}$ has a local minimum when $\Lambda_{0}$ is from the swampland. Table 3 and 4 summarize the values of $\Lambda_{0-\text { crit }}$ for all the cases of approximation in consideration. In [15, it is shown that $V(\phi)$ is a monotonically decreasing quintessence potential if $\Lambda_{0}$ is negative or from the string landscape while $V(\phi)$ is a meta-stable de Sitter one if $\Lambda_{0}$ is positive or from the swampland when $\Lambda_{e f f}$ is simulated by a scalar quintessence field potential $V(\phi)$. The dependence of $\Lambda_{0 \text {-crit }}$ on different values of $a_{0} H_{0}$ in both the cases of $K=+1$ and $K=-1$ is investigated in this paper and the numerical results are shown in Table 3 and 4 . A specific example solution of the $\Lambda_{\text {eff }}$ evolution pattern transition with the dependence on $\Lambda_{0}$ is shown in Figure 7. Figure 5 shows that $\Lambda_{\min }$ is positive if $a_{0} H_{0}$ takes a value less than 2 in the case of $K=+1$, i.e. $\Lambda_{\min }>\Lambda_{\max }$ so that $\Lambda_{0 \text {-crit }}$ does not make any sense in the case of $K=+1$ and $a_{0} H_{0} \geq 2$. 


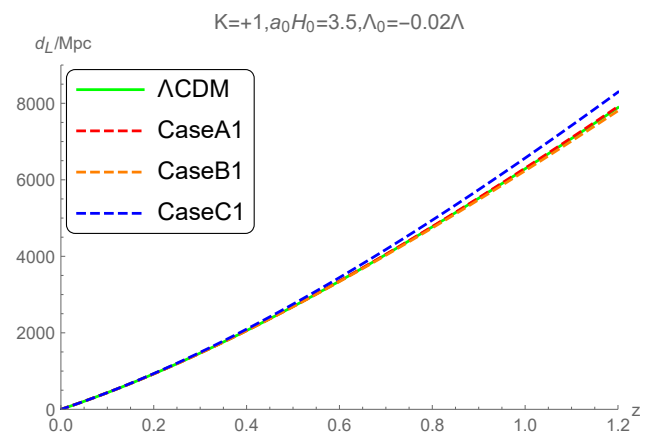

(a)

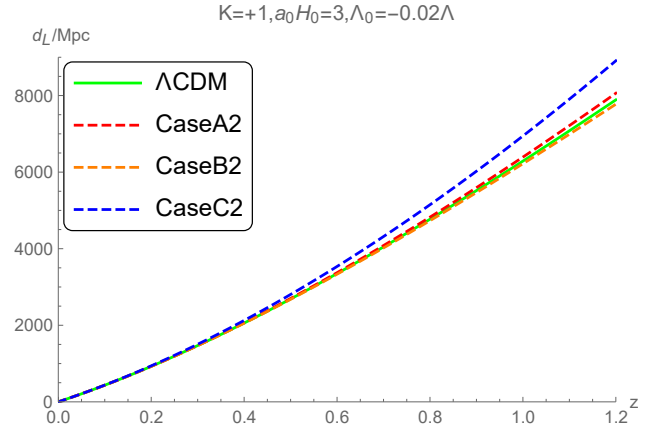

(c)

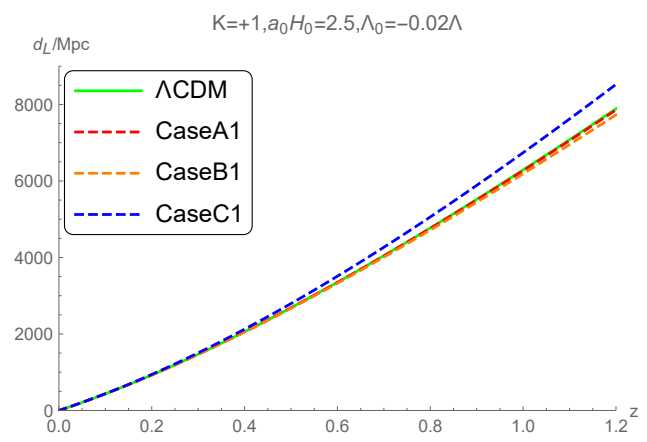

(e)

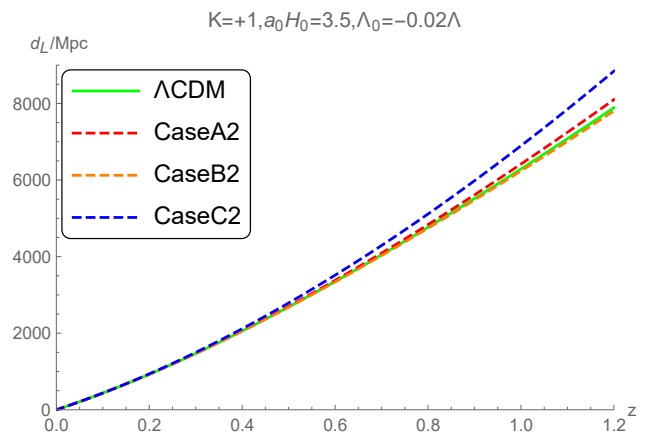

(b)

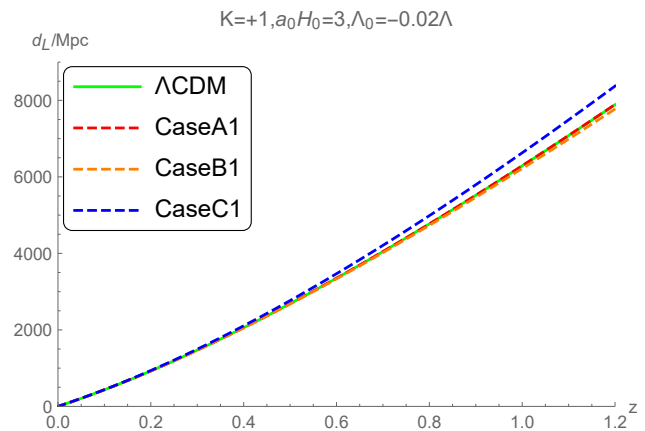

(d)

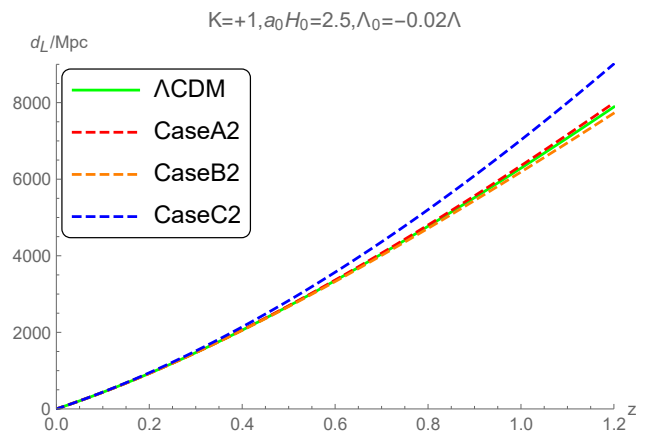

(f)

Figure 4: the luminosity disyance evolves with the redshift when $K=+1, w_{0}=-1, \Lambda_{0}=-0.02 \Lambda$. 
Table 1: The value of $\Lambda_{\max }$ when $K=+1$

\begin{tabular}{|c|c|c|c|c|}
\hline \multicolumn{5}{|c|}{$K=+1$} \\
\hline$\Lambda_{\max }$ & $\begin{array}{l}a_{0} H_{0}=2 \\
\left(\Omega_{k}=-0.25\right)\end{array}$ & $\begin{array}{l}a_{0} H_{0}=2.5 \\
\left(\Omega_{k}=-0.16\right)\end{array}$ & $\begin{array}{l}a_{0} H_{0}=3 \\
\left(\Omega_{k}=-0.11\right)\end{array}$ & $\begin{array}{l}a_{0} H_{0}=3.5 \\
\left(\Omega_{k}=-0.08\right)\end{array}$ \\
\hline CaseA1 & 0.0008 & 0.0007 & 0.0007 & 0.0006 \\
\hline CaseA2 & 0.0012 & 0.0014 & 0.0015 & 0.0015 \\
\hline CaseB1 & 0.0008 & 0.0007 & 0.0007 & 0.0006 \\
\hline CaseB2 & 0.0012 & 0.0014 & 0.0015 & 0.0016 \\
\hline $\begin{array}{l}\text { CaseC1 } \\
\left(w_{0}=-1\right)\end{array}$ & 0.0008 & 0.0007 & 0.0007 & 0.0006 \\
\hline $\begin{array}{l}\text { CaseC2 } \\
\left(w_{0}=-1\right)\end{array}$ & 0.0012 & 0.0015 & 0.0015 & 0.0016 \\
\hline $\begin{array}{l}\text { CaseC1 } \\
\left(w_{0}=-8 / 9\right)\end{array}$ & 0.0008 & 0.0007 & 0.0007 & 0.0006 \\
\hline $\begin{array}{l}\text { CaseC2 } \\
\left(w_{0}=-8 / 9\right)\end{array}$ & 0.0013 & 0.0016 & 0.0017 & 0.0018 \\
\hline $\begin{array}{l}\text { CaseC1 } \\
\left(w_{0}=-7 / 9\right)\end{array}$ & 0.0008 & 0.0007 & 0.0007 & 0.0006 \\
\hline $\begin{array}{l}\text { CaseC2 } \\
\left(w_{0}=-7 / 9\right)\end{array}$ & 0.0013 & 0.0016 & 0.0018 & 0.0019 \\
\hline $\begin{array}{l}\text { CaseC1 } \\
\left(w_{0}=-2 / 3\right)\end{array}$ & 0.0008 & 0.0007 & 0.0006 & 0.0006 \\
\hline $\begin{array}{l}\text { CaseC2 } \\
\left(w_{0}=-2 / 3\right)\end{array}$ & 0.0014 & 0.0018 & 0.002 & 0.0022 \\
\hline $\begin{array}{l}\text { CaseC1 } \\
\left(w_{0}=-1 / 3\right)\end{array}$ & 0.0008 & 0.0006 & 0.0006 & 0.0006 \\
\hline $\begin{array}{l}\text { CaseC2 } \\
\left(w_{0}=-1 / 3\right)\end{array}$ & 0.0025 & 0.0046 & 0.0056 & 0.0062 \\
\hline
\end{tabular}

Table 2: The value of $\Lambda_{\max }$ when $K=-1$

\begin{tabular}{|c|c|c|c|c|c|}
\hline \multicolumn{6}{|c|}{$K=-1$} \\
\hline$\Lambda_{\max }$ & $\begin{array}{l}a_{0} H_{0}=1.5 \\
\left(\Omega_{k}=0.44\right)\end{array}$ & $\begin{array}{l}a_{0} H_{0}=2 \\
\left(\Omega_{k}=0.25\right)\end{array}$ & $\begin{array}{l}a_{0} H_{0}=2.5 \\
\left(\Omega_{k}=0.16\right)\end{array}$ & $\begin{array}{l}a_{0} H_{0}=3 \\
\left(\Omega_{k}=0.11\right)\end{array}$ & $\begin{array}{l}a_{0} H_{0}=3.5 \\
\left(\Omega_{k}=0.08\right)\end{array}$ \\
\hline CaseA1 & 0.0004 & 0.0005 & 0.0005 & 0.0005 & 0.0005 \\
\hline CaseA2 & 0.0023 & 0.002 & 0.0019 & 0.0018 & 0.0018 \\
\hline CaseB1 & 0.0004 & 0.0004 & 0.0005 & 0.0005 & 0.0005 \\
\hline CaseB2 & 0.0023 & 0.002 & 0.0019 & 0.0019 & 0.0018 \\
\hline $\begin{array}{l}\text { CaseC1 } \\
\left(w_{0}=-1\right)\end{array}$ & 0.0003 & 0.0004 & 0.0005 & 0.0005 & 0.0005 \\
\hline $\begin{array}{l}\text { CaseC2 } \\
\left(w_{0}=-1\right)\end{array}$ & 0.015 & 0.0027 & 0.0023 & 0.0021 & 0.002 \\
\hline $\begin{array}{l}\text { CaseC1 } \\
\left(w_{0}=-8 / 9\right)\end{array}$ & 0.0003 & 0.0004 & 0.0005 & 0.0005 & 0.0005 \\
\hline $\begin{array}{l}\text { CaseC2 } \\
\left(w_{0}=-8 / 9\right)\end{array}$ & 0.0033 & 0.0033 & 0.0026 & 0.0024 & 0.0023 \\
\hline $\begin{array}{l}\text { CaseC1 } \\
\left(w_{0}=-7 / 9\right)\end{array}$ & 0.0003 & 0.0004 & 0.0005 & 0.0005 & 0.0005 \\
\hline $\begin{array}{l}\text { CaseC2 } \\
\left(w_{0}=-7 / 9\right)\end{array}$ & 0.0032 & 0.0032 & 0.0032 & 0.0028 & 0.0026 \\
\hline $\begin{array}{l}\text { CaseC1 } \\
\left(w_{0}=-2 / 3\right)\end{array}$ & 0.0003 & 0.0004 & 0.0004 & 0.0004 & 0.0005 \\
\hline $\begin{array}{l}\text { CaseC2 } \\
\left(w_{0}=-2 / 3\right)\end{array}$ & 0.0185 & 0.0091 & 0.0054 & 0.0037 & 0.0031 \\
\hline $\begin{array}{l}\text { CaseC1 } \\
\left(w_{0}=-1 / 3\right)\end{array}$ & 0.001 & 0.0005 & 0.0005 & 0.0005 & 0.0005 \\
\hline $\begin{array}{l}\text { CaseC2 } \\
\left(w_{0}=-1 / 3\right)\end{array}$ & 0.0147 & 0.0119 & 0.0105 & 0.0097 & 0.0092 \\
\hline
\end{tabular}




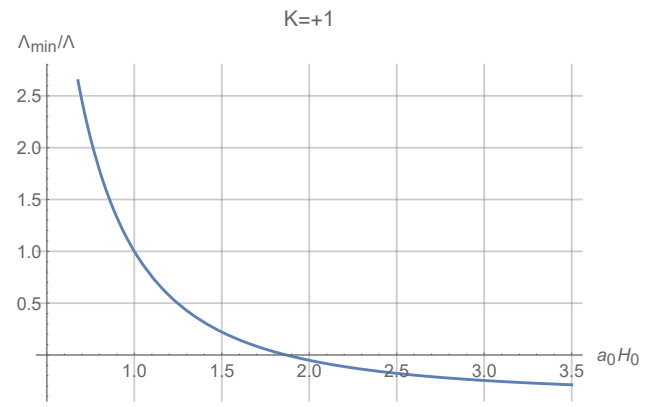

(a)

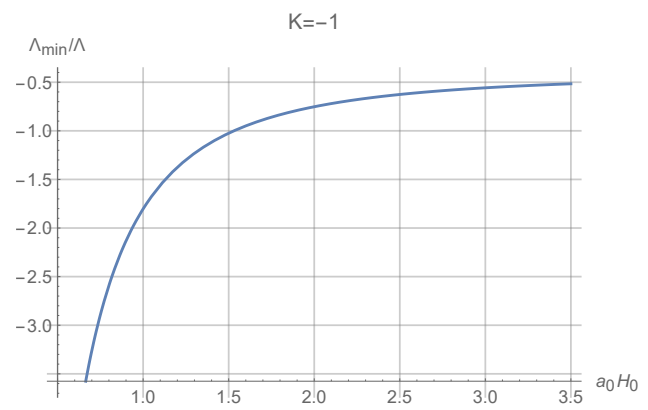

(b)

Figure 5: The evolution of the $\Lambda_{\min }$ versus $a_{0} H_{0}$

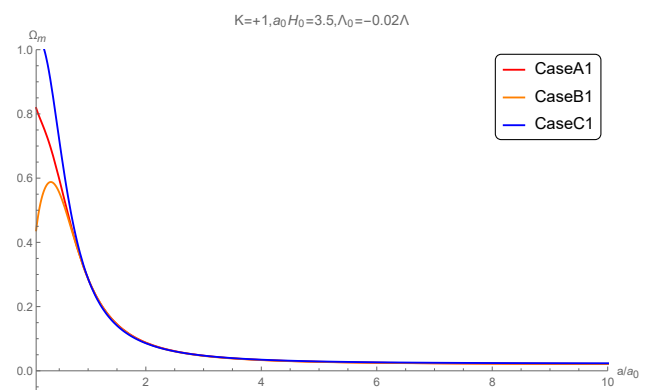

(a)

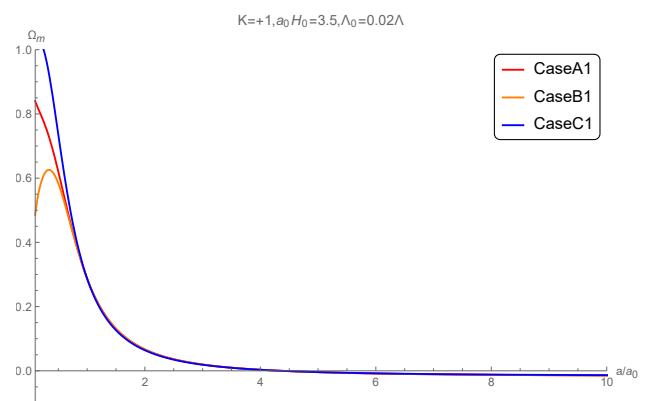

(b)

Figure 6: The evolution of the matter density $\Omega_{M}$ versus the scale factor

When the $w_{0}$ value of Case C2 is greater than $-\frac{8}{9}$, the $\Lambda_{\text {eff }}$ shows a quintessence like potential that decreases monotonically over time without a local minimum, i.e. there is no solution for $\Lambda_{0-\text { crit }}$. It is possible that the behavior is caused by the fixed value of $w_{0}$ other than an evolved one in the equation of state for dark partner. A similar conclusion is reached based on the large-scale Lorentz violation model without spatial curvature in [13, 14. When $w_{0}>-\frac{8}{9}$, the model prediction on luminosity distance modulus versus redshift curve of Case $\mathrm{C} 2$ does not match the observation one in case of $K=0$ while the prediction on evolution luminosity distance modulus is compatible with observation one in case of $K \neq 0$. The spatial non-flat Case $\mathrm{C} 2$ with $w_{0}>-\frac{8}{9}$ can not be excluded by observation just like spatial flat case as in [13, 14]. However, we find that the $\Lambda_{0-\text { crit }}$ of Case $\mathrm{C}$ is always greater than $\Lambda_{\max }$ value when $w_{0}>-1$ from Tables 1, 2, 3 and 4, i.e. the solution of $\Lambda_{0-\text { crit }}$ for Case C does not exist within the range of values that can be taken for $\Lambda_{0}$ when $w_{0}>-1$. Comparisons of the luminosity distance $d_{L}$ curve versus redshift $z$ among three models of approximation and $\Lambda$ CDM model are presented in Figure 8 to Figure 13 .

The Figures 8 to 13 show that the predictions of the luminosity distance modulus of the three cases of approximation models for different spatial curvature values with the restricted range of $\Lambda-0$ values are all compatible with observational data within the error range. That is, the luminosity distance observations are not efficiency in choosing the models.

The evolution of $\Omega_{\Lambda}=\frac{\Lambda}{3 H^{2}}$ in $\Lambda$ CDM model, $\Omega_{e f f}(K=0)$ in the large-scale Lorentz violation model without spatial curvature and $\Omega_{e f f}(K= \pm 1)$ in the large-scale Lorentz violation model with spatial curvature are presented in Figure 14 which shows that the evolution behaviors of $\Omega_{\Lambda}, \Omega_{\text {eff }}(K=0)$ and $\Omega_{e f f}(K= \pm 1)$ are similar, so their contributions are degenerate in result.

It is shown in Figure 15 that the evolution of the energy density of the spatial curvature $\Omega_{K}=-\frac{K}{a^{2} H^{2}}$ versus scale factor $a$ based on the modified Friedmann equations for both the cases of $K=+1$ and $K=-1$. The maximum and minimum of $\Omega_{K}$ can also be obtained from the evolution of $\Omega_{K}$. When $K=+1$, the Case $\mathrm{C} 1$ has the minimum curvature energy density $\Omega_{K}=-0.34$, while the Case A1 has 
Table 3: The value of $\Lambda_{0-\text { crit }}$ in case of $K=+1$

\begin{tabular}{|c|c|c|c|c|}
\hline \multicolumn{4}{|c|}{$K=+1$} & \multirow[t]{2}{*}{$K=0$} \\
\hline$\Lambda_{0-c r i t}$ & $a_{0} H_{0}=2.5$ & $a_{0} H_{0}=3$ & $a_{0} H_{0}=3.5$ & \\
\hline CaseA1 & -0.072 & -0.064 & -0.059 & 0.05 \\
\hline CaseA2 & -0.14 & -0.0159 & -0.167 & -0.18 \\
\hline CaseB1 & -0.094 & -0.083 & -0.078 & -0.066 \\
\hline CaseB2 & -0.154 & -0.176 & -0.187 & -0.2144 \\
\hline $\begin{array}{l}\text { CaseC } 1 \\
\left(w_{0}=-1\right)\end{array}$ & 0 & 0 & 0 & 0 \\
\hline $\begin{array}{l}\text { CaseC2 } \\
\left(w_{0}=-1\right)\end{array}$ & 0 & 0 & 0 & 0 \\
\hline $\begin{array}{l}\text { CaseC } 1 \\
\left(w_{0}=-8 / 9\right)\end{array}$ & 0.162 & 0.152 & 0.146 & 0.119 \\
\hline $\begin{array}{l}\text { CaseC2 } \\
\left(w_{0}=-8 / 9\right)\end{array}$ & 0.09 & 0.086 & 0.083 & 0.075 \\
\hline $\begin{array}{l}\text { CaseC1 } \\
\left(w_{0}=-7 / 9\right)\end{array}$ & none & none & none & none \\
\hline $\begin{array}{l}\text { CaseC2 } \\
\left(w_{0}=-7 / 9\right)\end{array}$ & 0.173 & 0.164 & 0.164 & 0.152 \\
\hline $\begin{array}{l}\text { CaseC1 } \\
\left(w_{0}=-2 / 3\right)\end{array}$ & none & none & none & none \\
\hline $\begin{array}{l}\text { CaseC2 } \\
\left(w_{0}=-2 / 3\right)\end{array}$ & 0.246 & 0.235 & 0.228 & 0.225 \\
\hline $\begin{array}{l}\text { CaseC1 } \\
\left(w_{0}=-1 / 3\right)\end{array}$ & none & none & none & none \\
\hline $\begin{array}{l}\text { CaseC2 } \\
\left(w_{0}=-1 / 3\right)\end{array}$ & 0.354 & 0.368 & 0.375 & 0.397 \\
\hline
\end{tabular}

Table 4: The value of $\Lambda_{0-\text { crit }}$ in case of $K=-1$

\begin{tabular}{|c|c|c|c|c|c|c|}
\hline \multicolumn{6}{|c|}{$K=-1$} & \multirow[t]{2}{*}{$K=0$} \\
\hline$\Lambda_{0-c r i t}$ & $a_{0} H_{0}=-1.5$ & $a_{0} H_{0}=-2$ & $a_{0} H_{0}=-2.5$ & $a_{0} H_{0}=-3$ & $a_{0} H_{0}=-3.5$ & \\
\hline CaseA1 & -0.023 & -0.033 & -0.038 & -0.041 & -0.044 & -0.05 \\
\hline CaseA2 & -0.214 & -0.208 & -0.203 & -0.198 & -0.195 & -0.187 \\
\hline CaseB1 & -0.03 & -0.042 & -0.049 & -0.053 & -0.056 & -0.066 \\
\hline CaseB2 & -0.284 & -0.262 & -0.248 & -0.239 & -0.233 & -0.2144 \\
\hline $\begin{array}{l}\text { CaseC1 } \\
\left(w_{0}=-1\right)\end{array}$ & 0 & 0 & 0 & 0 & 0 & 0 \\
\hline $\begin{array}{l}\text { CaseC2 } \\
\left(w_{0}=-1\right)\end{array}$ & 0 & 0 & 0 & 0 & 0 & 0 \\
\hline $\begin{array}{l}\text { CaseC1 } \\
\left(w_{0}=-8 / 9\right)\end{array}$ & 0.05 & 0.086 & 0.102 & 0.107 & 0.115 & 0.119 \\
\hline $\begin{array}{l}\text { CaseC2 } \\
\left(w_{0}=-8 / 9\right)\end{array}$ & none & 0.03 & 0.052 & 0.059 & 0.064 & 0.075 \\
\hline $\begin{array}{l}\text { CaseC1 } \\
\left(w_{0}=-7 / 9\right)\end{array}$ & none & none & none & none & none & none \\
\hline $\begin{array}{l}\text { CaseC2 } \\
\left(w_{0}=-7 / 9\right)\end{array}$ & none & 0.079 & 0.104 & 0.118 & 0.131 & 0.152 \\
\hline $\begin{array}{l}\text { CaseC1 } \\
\left(w_{0}=-2 / 3\right)\end{array}$ & none & none & none & none & none & none \\
\hline $\begin{array}{l}\text { CaseC2 } \\
\left(w_{0}=-2 / 3\right)\end{array}$ & 0.03 & 0.15 & 0.173 & 0.189 & 0.198 & 0.225 \\
\hline $\begin{array}{l}\text { CaseC1 } \\
\left(w_{0}=-1 / 3\right)\end{array}$ & none & none & none & none & none & none \\
\hline $\begin{array}{l}\text { CaseC2 } \\
\left(w_{0}=-1 / 3\right)\end{array}$ & 0.203 & 0.25 & 0.278 & 0.309 & 0.343 & 0.397 \\
\hline
\end{tabular}




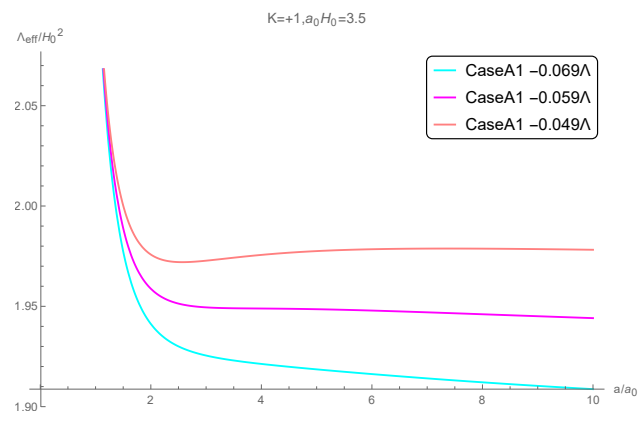

Figure 7: The evolution pattern of $\Lambda_{\text {eff }}$ versus scale factor shifts from a monotonically decreasing quintessence type to a local minimal type with the dependence on $\Lambda_{0}$

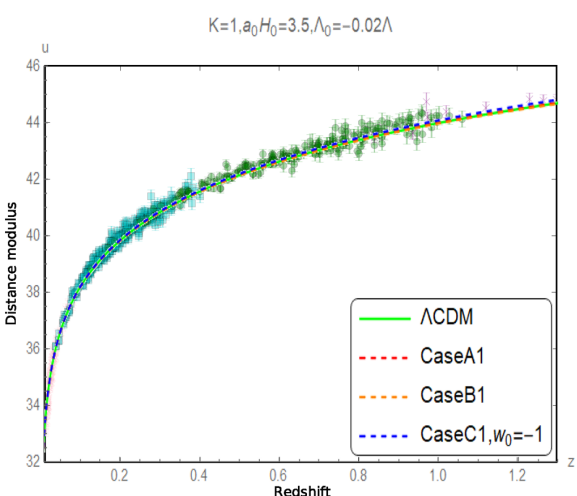

(a)

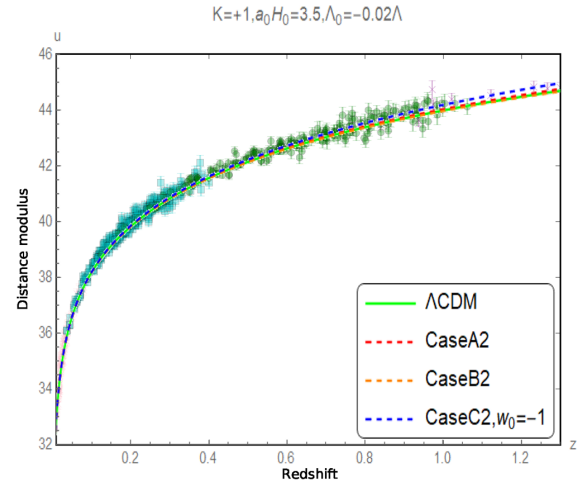

(b)

Figure 8: Comparison of the measured distance modulus with the corresponding predicted values when $K=+1, \Lambda_{0}=-0.02 \Lambda$ and $a_{0} H_{0}=3.5$

the maximum one with $\Omega_{K}=0.6$ when $K=-1$. The evolution of the absolute values of the curvature energy densities always increase at first stage then decrease to zero after reaching the maximum. Figures 1 to 3 can also supply the evolution tendency of the absolute values of the curvature densities, where the evolution of $H$ is decreasing and $a^{2} H^{2}$ has a minimum value during evolution.

The evolution of the acceleration of the universe expansion $\ddot{a}$ can also be obtained by the modified Friedmann equations and the approximations considered. The evolution of $\ddot{a}$ versus scale factor $a$ can be found in Figure 16, from which it can be roughly obtained that the expansion of the universe is decelerating before the cosmic scale factor reaches $0.5 a_{0}$ and the universe becomes accelerating afterwards. The conclusion is consistent with the observed universe experiencing the transition from decelerating expansion to accelerating one, thus supplying a further side proof for the confidence level of the large scale Lorentz violation model with non-vanishing spatial curvature.

\section{Summary and Outlook}

The cause of the $H_{0}$ tension between high and low redshift measurements is so far unknown. Many solutions to $H_{0}$ tension have been proposed. It was suggested that this was due to systematic error, however these claims were quickly disproved [16-19]. The gravitational waves from the binary neutron star (BNS) merger may be able to make independent measurements of $H_{0}$ [20, although future observations should be able to reduce the error 21 26. However, this current measurement method has a large error (due to being a single event) and therefore does not resolve the tension between $H_{0}$ measurements. Furthermore, as one of the simplest alternatives to the $\Lambda \mathrm{CDM}$ model: quintessence fields with smooth potential energy, rolling slowly or at moderate velocity in the non-flat FRW universe, are expected to 


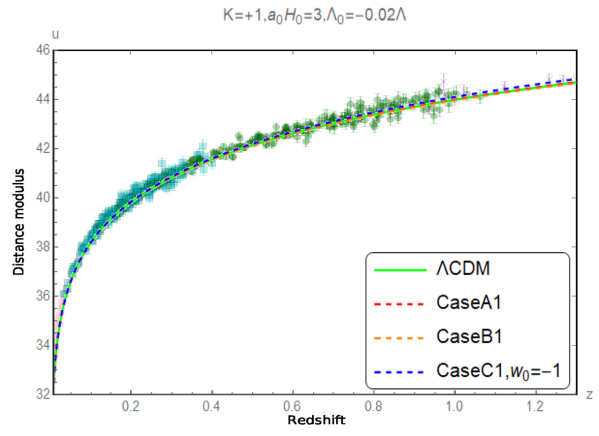

(a)

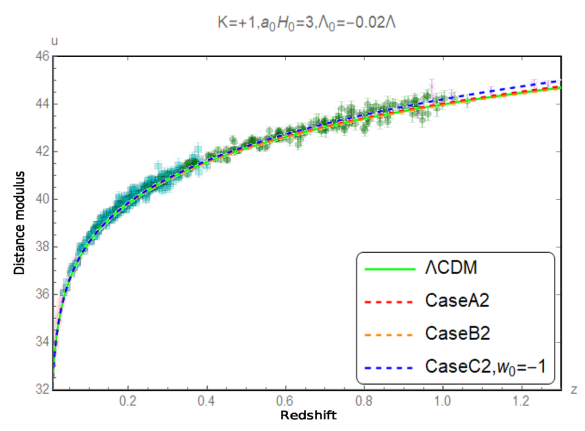

(b)

Figure 9: Comparison of the measured distance modulus with the corresponding predicted values when $K=+1, \Lambda_{0}=-0.02 \Lambda$ and $a_{0} H_{0}=3$

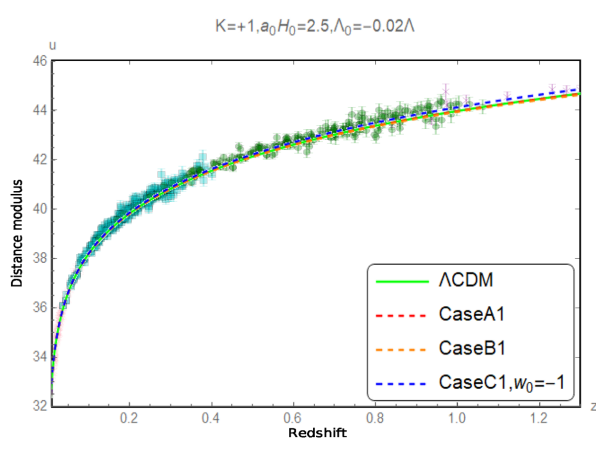

(a)

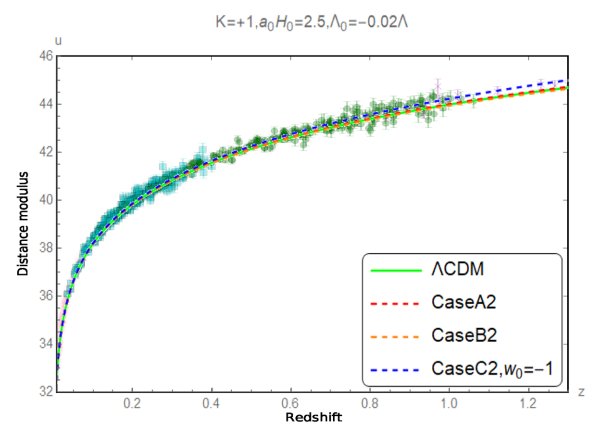

(b)

Figure 10: Comparison of the measured distance modulus with the corresponding predicted values when $K=+1, \Lambda_{0}=-0.02 \Lambda$ and $a_{0} H_{0}=2.5$

resolve the tension between Hubble constant measurements. But after combining the latest $\mathrm{CMB}, \mathrm{SNe}$ and BAO data, it was found that this model does not reduce the Hubble constant tension 27.

Literature 28 presents a new solution to the Hubble constant problem. The solution is based on the Simsilun simulation (relativistic simulation of the large scale structure of the Universe) with the ray-tracing algorithm implemented, within the Simsilun simulation relativistic and nonlinear evolution of cosmic structures leads to the phenomenon of emerging spatial curvature, where the mean spatial curvature evolves from spatial flatness of the early universe towards slightly curved present-day universe. The deduced Hubble constant, $H_{0}=(68.1 \pm 2.0) \mathrm{km} /(\mathrm{s} \cdot \mathrm{Mpc})$, alleviates the tension between the CMB and distance ladder measurements of the Hubble constant and it is argued that the $H_{0}$ tension is a manifestation of rigidity of the FLRW geometry.

At the current stage, the existence of spatial curvature seems to be a viable solution to the Hubble constant problem. In fact, we can also counter that the tension is an indirect evidence of the emerging spatial curvature. From the point of view of astronomical observations, no direct measurement of spatial curvature has yet been made at low redshifts (the constraint obtained is also only the result of fitting FLRW to the data, which is not equivalent to a direct measurement).

In conclusion, although the large scale Lorentz violation model with non-vanishing spatial curvature has one more cosmological scale contortion parameter than the $\Lambda \mathrm{CDM}$ model with non-vanishing spatial curvature, the inputs are well constrained in a variety of ways, and the theoretical predictions of distance modulus, cosmic expansion acceleration, etc. are in good agreement with observations.

The temperature fluctuation in CMB power spectra can fix the cosmological parameters of $\Lambda$ CDM model strictly. The Hubble constant predicted by the large scale Lorentz violation with non-vanishing 


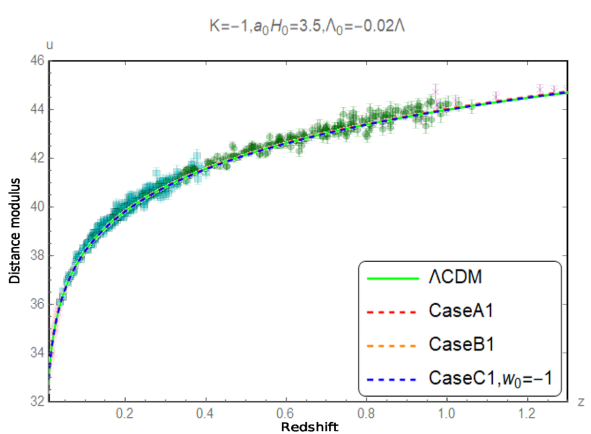

(a)

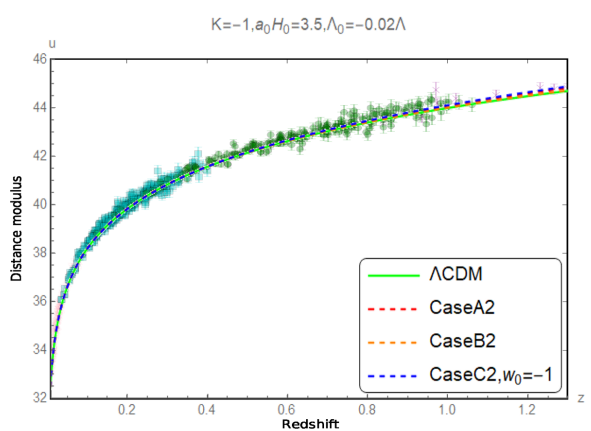

(b)

Figure 11: Comparison of the measured distance modulus with the corresponding predicted values when $K=-1, \Lambda_{0}=-0.02 \Lambda$ and $a_{0} H_{0}=3.5$

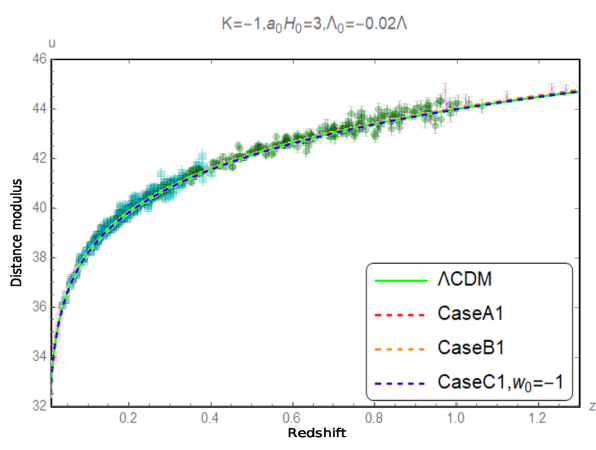

(a)

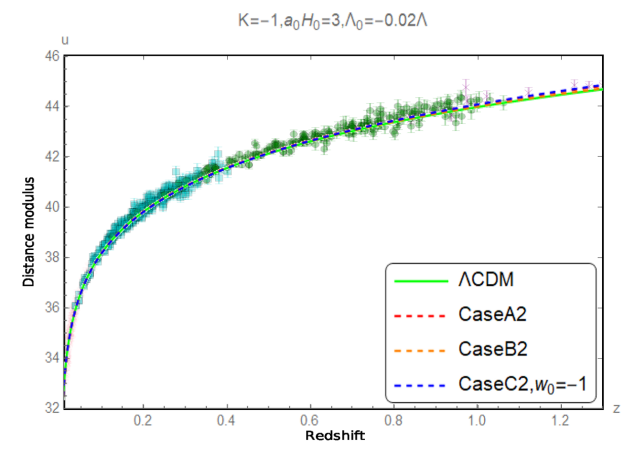

(b)

Figure 12: Comparison of the measured distance modulus with the corresponding predicted values when $K=-1, \Lambda_{0}=-0.02 \Lambda$ and $a_{0} H_{0}=3$

spatial curvature is also constrained by the CMB data and be compared with one predicted by CMB data based on $\Lambda \mathrm{CDM}$ model to evaluate the improvement on the $H_{0}$ tension problem by the large scale Lorentz violation with non-vanishing spatial curvature model. The cosmological parameters of the specific cosmological model such as $\Lambda$ CDM model can be obtained from CMB data by the numerical software like Code for Anisotropies in the Microwave Background(CAMB). It is pointed out that the spatial flatness assumption may be responsible for the $H_{0}$ tension, the non-vanishing spatial curvature is an alternative choice. This paper provides a viable solution to $H_{0}$ tension by investigating the constrain on the spatial curvature provided by the large scale Lorentz violation cosmological model with spatial curvature. We find that the non-vanishing of the aptial curvature is compatible with the present observation. The model provides an viable premise to solve the $H_{0}$ tension problem by the spatial non-flat cosmological model. The numerical software like CAMB needs to be extended to the model with non-trivial contortion distribution to present the $H_{0}$ value in this kind of models.

\section{Acknowledgment}

This work is supported by the National Natural Science Foundation of China, under Grant No. 11775080 and Grant No. 11865016. 


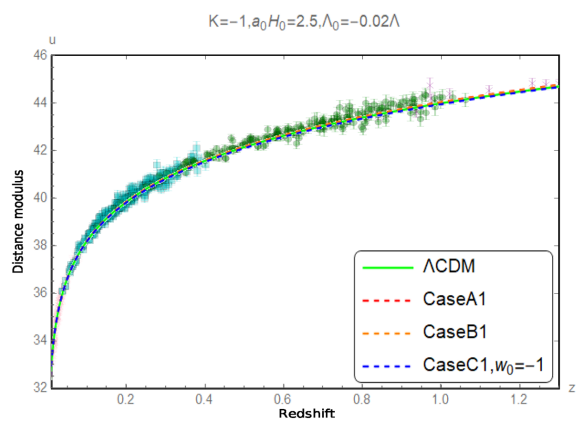

(a)

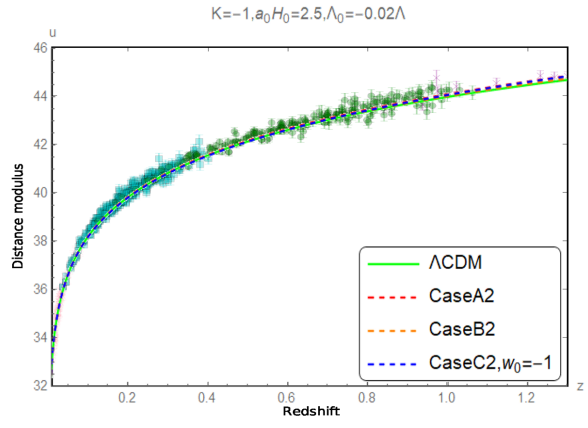

(b)

Figure 13: Comparison of the measured distance modulus with the corresponding predicted values when $K=-1, \Lambda_{0}=-0.02 \Lambda$ and $a_{0} H_{0}=2.5$

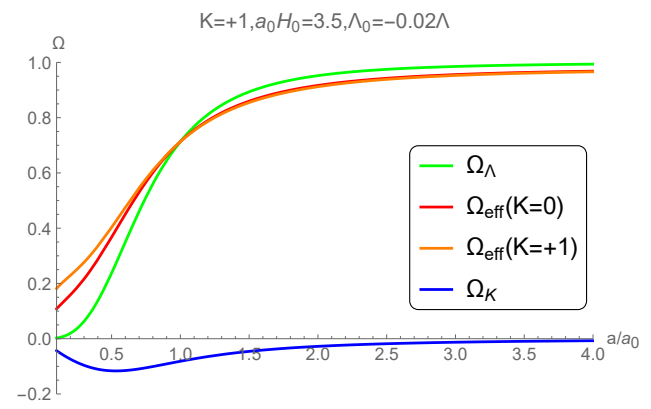

(a)

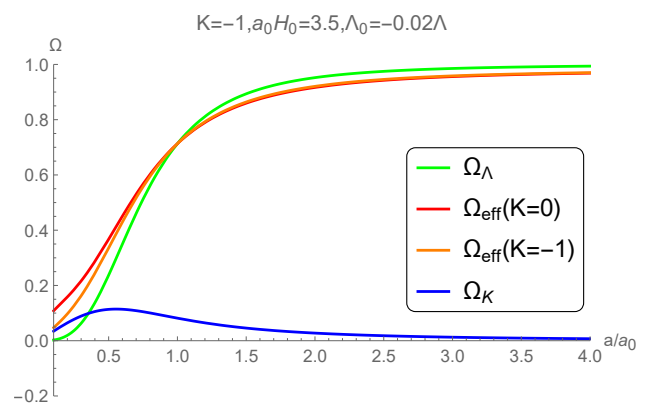

(b)

Figure 14: The evolution of $\Omega_{\Lambda}, \Omega_{e f f}(K=0), \Omega_{e f f}(K= \pm 1)$ and $\Omega_{k}$

\section{References}

[1] Planck Collaboration, N. Aghanim et al., "Planck 2018 results. VI. Cosmological parameters," Astron. Astrophys. 641 (2020) A6, arXiv:1807.06209 [astro-ph.C0], [Erratum:

Astron.Astrophys. 652, C4 (2021)].

[2] P. A. Ade et al., "Planck 2013 results. I. Overview of products and scientific results," Astronomy \& Astrophysics 571 (2014) A1.

[3] A. G. Riess et al., "Milky Way Cepheid Standards for Measuring Cosmic Distances and Application to Gaia DR2: Implications for the Hubble Constant," Astrophys. J. 861 no. 2, (2018) 126, arXiv:1804.10655 [astro-ph.CO].

[4] J. M. Virey, D. Talon-Esmieu, A. Ealet, P. Taxil, and A. Tilquin, "On the determination of curvature and dynamical Dark Energy," JCAP 12 (2008) 008, arXiv:0802.4407 [astro-ph].

[5] Y. Wang and P. Mukherjee, "Observational Constraints on Dark Energy and Cosmic Curvature," Phys. Rev. D 76 (2007) 103533, arXiv:astro-ph/0703780.

[6] C. Clarkson, M. Cortes, and B. A. Bassett, "Dynamical Dark Energy or Simply Cosmic Curvature?," JCAP 08 (2007) 011, arXiv: astro-ph/0702670.

[7] A. Rest et al., "Cosmological Constraints from Measurements of Type Ia Supernovae discovered during the first 1.5 yr of the Pan-STARRS1 Survey," Astrophys. J. 795 no. 1, (2014) 44. arXiv: 1310.3828 [astro-ph.CO]. 


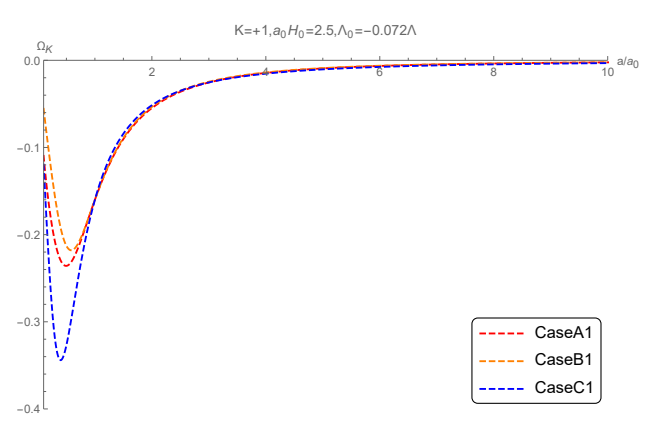

(a)

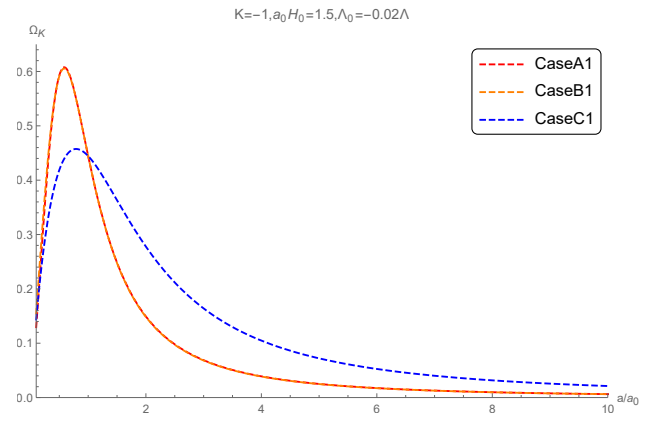

(b)

Figure 15: The $\Omega_{K}$ evolves with the scale factor.

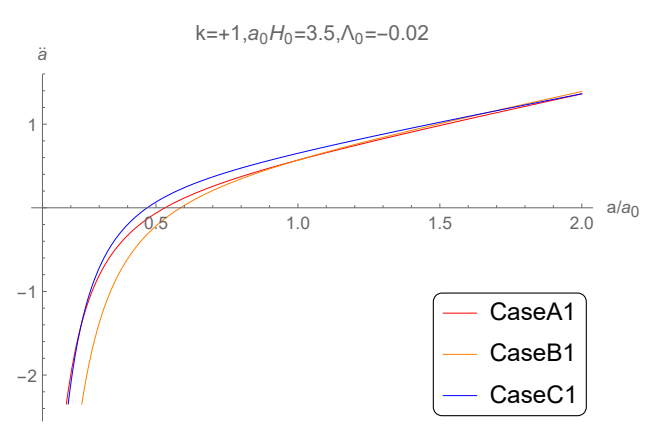

(a)

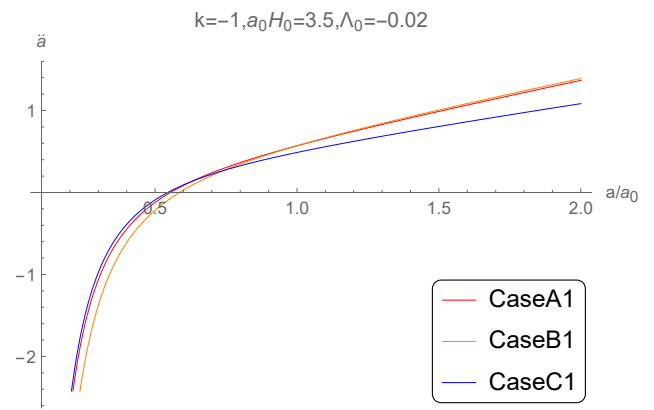

(b)

Figure 16: The evolution of $\ddot{a}$ versus the scale factor

[8] S. Kumar, "Consistency of nonflat $\Lambda$ CDM model with the new result from BOSS," Phys. Rev. D 92 no. 10, (2015) 103512, arXiv:1507.04684 [gr-qc].

[9] E. Di Valentino, A. Melchiorri, and J. Silk, "Planck evidence for a closed Universe and a possible crisis for cosmology," Nature Astron. 4 no. 2, (2019) 196-203, arXiv:1911.02087 [astro-ph.CD].

[10] E. Di Valentino, A. Melchiorri, and J. Silk, "Investigating Cosmic Discordance," Astrophys. J. Lett. 908 no. 1, (2021) L9, arXiv:2003.04935 [astro-ph.CO].

[11] Q.-G. Huang and M. Li, "The Holographic dark energy in a non-flat universe," JCAP 08 (2004) 013, arXiv: astro-ph/0404229

[12] J. Shen and X. Xue, "Large Scale Lorentz Violation Gravity and Dark Energy," in Proceedings, 28th International Symposium on Lepton Photon Interactions at High Energies (LP17):

Guangzhou (Guangdong), China, August 7-12, 2017, W. Wang and Z.-z. Xing, eds., pp. 459-475. 2020. arXiv:1802.03502 [gr-qc].

[13] H. Zhai, J. Shen, and X. Xue, "Effective quintessence from string landscape," Acta Phys. Sin. 68 no. $13,(2019) 139501$

[14] H. Zhai, J. Shen, and X. Xue, "Uplifting of AdS type to quintessence-like potential induced by frozen large-scale Lorentz violation," Chin. Phys. C 44 no. 8, (2020) 085101, arXiv:1906.11860 [hep-th].

[15] Q. Li, J. Li, Y. Zhou, and X. Xue, "The Effective Potential Originating from Swampland and the Non-trivial Brans-Dicke Coupling," Chin. Phys. C 44 no. 10, (2020) 105108, arXiv: 2003.09121 [gr-qc]. 
[16] T. Shanks, L. Hogarth, and N. Metcalfe, "Gaia Cepheid parallaxes and 'Local Hole' relieve $H_{0}$ tension," Mon. Not. Roy. Astron. Soc. 484 no. 1, (2019) L64-L68, arXiv:1810.02595 [astro-ph.CO].

[17] A. G. Riess, S. Casertano, D. Kenworthy, D. Scolnic, and L. Macri, "Seven Problems with the Claims Related to the Hubble Tension in arXiv:1810.02595," arXiv:1810.03526 [astro-ph.C0].

[18] R. von Marttens, V. Marra, L. Casarini, J. E. Gonzalez, and J. Alcaniz, "Null test for interactions in the dark sector," Phys. Rev. D 99 no. 4, (2019) 043521, arXiv:1812.02333 [astro-ph.C0].

[19] C. A. P. Bengaly, U. Andrade, and J. S. Alcaniz, "How does an incomplete sky coverage affect the Hubble Constant variance?," Eur. Phys. J. C 79 no. 9, (2019) 768, arXiv:1810.04966 [astro-ph.CO].

[20] LIGO Scientific, Virgo Collaboration, B. P. Abbott et al., "GW170817: Observation of Gravitational Waves from a Binary Neutron Star Inspiral," Phys. Rev. Lett. 119 no. 16, (2017) 161101, arXiv:1710.05832 [gr-qc].

[21] LIGO Scientific, Virgo Collaboration, M. Fishbach et al., "A Standard Siren Measurement of the Hubble Constant from GW170817 without the Electromagnetic Counterpart," Astrophys. J. Lett. 871 no. 1, (2019) L13, arXiv:1807.05667 [astro-ph.C0].

[22] D. J. Mortlock, S. M. Feeney, H. V. Peiris, A. R. Williamson, and S. M. Nissanke, "Unbiased Hubble constant estimation from binary neutron star mergers," Phys. Rev. D 100 no. 10, (2019) 103523, arXiv:1811.11723 [astro-ph.CO]

[23] S. M. Feeney, H. V. Peiris, A. R. Williamson, S. M. Nissanke, D. J. Mortlock, J. Alsing, and D. Scolnic, "Prospects for resolving the Hubble constant tension with standard sirens," Phys. Rev. Lett. 122 no. 6, (2019) 061105, arXiv:1802.03404 [astro-ph.C0]

[24] K. Hotokezaka, E. Nakar, O. Gottlieb, S. Nissanke, K. Masuda, G. Hallinan, K. P. Mooley, and A. T. Deller, "A Hubble constant measurement from superluminal motion of the jet in GW170817," Nature Astron. 3 no. 10, (2019) 940-944, arXiv:1806.10596 [astro-ph.C0]

[25] H.-Y. Chen, M. Fishbach, and D. E. Holz, "A two per cent Hubble constant measurement from standard sirens within five years," Nature 562 no. 7728, (2018) 545-547, arXiv:1712.06531 [astro-ph.CO].

[26] S. Vitale and H.-Y. Chen, "Measuring the Hubble constant with neutron star black hole mergers," Phys. Rev. Lett. 121 no. 2, (2018) 021303, arXiv:1804.07337 [astro-ph.C0].

[27] H. Miao and Z. Huang, "The $H_{0}$ Tension in Non-flat QCDM Cosmology," Astrophys. J. 868 no. 1, (2018) 20, arXiv:1803.07320 [astro-ph.CO].

[28] K. Bolejko, "Emerging spatial curvature can resolve the tension between high-redshift CMB and low-redshift distance ladder measurements of the Hubble constant," Phys. Rev. D 97 no. 10, (2018) 103529, arXiv:1712.02967 [astro-ph.CO] 